\title{
Absolutely classical spin states
}

\author{
F. Bohnet-Waldraff ${ }^{1,2}$, O. Giraud ${ }^{1}$, and D. Braun ${ }^{2}$ \\ ${ }^{1}$ LPTMS, CNRS, Univ. Paris-Sud, Université Paris-Saclay, 91405 Orsay, France \\ ${ }^{2}$ Institut für theoretische Physik, Universität Tübingen, 72076 Tübingen, Germany
}

\begin{abstract}
We introduce the concept of "absolutely classical" spin states, in analogy to absolutely separable states of bi-partite quantum systems. Absolutely classical states are states that remain classical under any unitary transformation applied to them. We investigate the maximum ball of absolutely classical states centered on the fully mixed state that can be inscribed into the set of classical states, and derive a lower bound for its radius as function of the total spin quantum number. The result is compared to the case of absolutely separable states.
\end{abstract}

PACS numbers: 03.65.Aa, 03.65.Ud, 03.67.-a

\section{INTRODUCTION}

The rise of quantum information technology has led to the need to classify and quantify the resources that ultimately enable a quantum advantage in certain computational, communicational, or metrological tasks. Most of the efforts have concentrated on classifying entanglement. Indeed, entanglement has been recognized to be necessary for e.g. computational speed-ups (at least for pure states) [1], quantum teleportation [2], super-dense coding [3], and quantum data hiding [4]. It can also be used for quantum key distribution [5], or for achieving enhanced precision in certain metrological applications [6]. Recently, it has been realized that other types of quantum mechanical correlations in the form of "quantum discord" exist that do not require entanglement but may still have useful applications [7].

Quantum entanglement necessarily requires at least bi-partite systems. However, even for a single system one can meaningfully ask to what extent a particular quantum state shows genuine quantum mechanical properties. In quantum optics such questions were investigated at least as early as the middle of last century. Quasi-probability distributions were introduced that allow one to distinguish "classical" quantum states from states that show genuine quantum effects such as enhanced quantum fluctuations of observables, or quantum interference, including multi-photon interference. An important role is played by coherent states of the radiation field, in which the quantum fluctuations of the field quadratures are minimal and evenly distributed over the canonical coordinates. Such states come as close as quantum mechanically possible to a point in classical phase space, and in general, one can consider as classical states of the radiation field that can be expressed as a convex sum (i.e. a classical mixture) of (projectors onto) coherent states [8, 9. Recently, these ideas were transferred to spin states, where $\mathrm{SU}(2)$ coherent states (introduced in [10]) play the role of the most classical pure states, and a mixed spin state is considered "classical" if it can be written as a statistical mixture of $\mathrm{SU}(2)$ coherent states 11. With this classification, all states of a spin- $1 / 2$ are classical as they can be expressed as classical mixtures of pure states with minimal quantum fluctuations. For a spin-1, there are genuinly nonclassical states, and necessary and sufficient conditions are known for classicality. These conditions can be used to explore analytically the geometry of quantum states [12] and provide a full analytical parametrization of the classical domain 13 . For higher values of $j$, one can find sufficient conditions for non-classicality from the positivity of correlation functions of spin observables [11, 14. By definition, the classical states form a convex set, and one can define a "quantumness" measure of a state as the distance from this state to the convex set of classical states, in analogy to geometric measures of entanglement [15, 16. Indeed, the two problems are related through the fact that spin- $j$ states can be also understood as states of $N=2 j$ spins- $1 / 2$ fully symmetric under permutation of particles, so that quantumness of a spin- $j$ is equivalent to entanglement of $N=2 j$ spins- $1 / 2$ in the fully symmetric sector of the Hilbert space of $N$ two-level systems [14.

Any measure of entanglement $E(\rho)$ is by definition invariant under local unitary operations. But one can also ask for states for which $E(\rho)$ is invariant under any unitary operation. In particular, states $\rho$ such that $E\left(U \rho U^{\dagger}\right)=0$ for all unitary operations $U$, called "absolutely separable" states, have attracted substantial interest [17 23]. Absolutely separable states have the property that no entanglement can be created from them, no matter how strongly and how long the corresponding particles interact. Conversely, for states which are not absolutely separable there is at least in principle the possibility that some entanglement be created from a common unitary evolution. The maximally mixed state $\rho_{0}$, which is proportional to the identity matrix, is obviously an absolutely separable state. As separable states form a closed set, there is a ball around $\rho_{0}$ such that all states within that ball are absolutely separable. Finding the largest radius of such a ball provides a sufficient condition for absolute separability; such a question was addressed in 20 .

In the present work, we ask an analogous question for 
quantumness: what are the states of a spin- $j$ that remain classical no matter what unitary evolution is applied to them? These states have the physical interpretation that no quantumness can be created from them in the course of any unitary time evolution, generated by an arbitrary, even time-dependent hamiltonian. We correspondingly call these states "absolutely classical". Alternatively, states that are not absolutely classical have the potential that in the course of some unitary evolution some quantumness may appear.

The aim of the paper is to provide a characterization of the set of absolutely classical states in terms of a maximum distance from the maximally mixed spin- $j$ state, such that any state closer to the fully mixed state is guaranteed to be classical. This distance is the maximal radius that a ball of classical states around the maximally mixed spin- $j$ state can have. We provide a lower bound for this maximum radius based on an expansion of the Glauber-Sudarshan $P$-function into spherical harmonics, and calculate a numerical approximation by randomly sampling a large number of states and mixing them with the fully mixed state until their quantumness vanishes. We start by defining the above concepts more precisely.

\section{ABSOLUTELY CLASSICAL STATES}

\section{A. Classical spin states}

Pure classical spin states were defined in [11] as $\mathrm{SU}(2)$ coherent states. This is motivated by the fact that these states have minimal possible uncertainty of the angular momentum operator J. Moreover, when the spin undergoes a unitary time evolution driven by a Hamiltonian linear in the components of $\mathbf{J}$, corresponding for example to a precession in a magnetic field, this minimal uncertainty property is conserved (similarly as what happens for field coherent states, see e.g. [24]). A spin- $j$ coherent state points in a well-defined direction $\mathbf{n}$ that we can parametrize with polar and azimuthal angles $\theta, \phi$ as $\mathbf{n}=(\sin \theta \cos \phi, \sin \theta \sin \phi, \cos \theta)$. In terms of the usual $|j, m\rangle$ basis states (i.e. eigenstates of $\mathbf{J}^{2}$ and $J_{z}$ with eigenvalues $j(j+1)$ and $m$, respectively, with $\hbar=1$ ), a spin- $j$ coherent state can be expanded as [25]

$|\alpha\rangle=\sum_{m=-j}^{j} \sqrt{\left(\begin{array}{c}2 j \\ j+m\end{array}\right)}\left(\cos \frac{\theta}{2}\right)^{j+m}\left(\sin \frac{\theta}{2} e^{-i \phi}\right)^{j-m}|j, m\rangle$,

with $\theta \in[0, \pi]$ and $\phi \in[0,2 \pi[$. By a stereographic projection $\alpha=e^{i \phi} \tan (\theta / 2)$, we can alternatively parametrize the spin- $j$ coherent states with a complex number $\alpha$. The $|\alpha\rangle$ form an overcomplete basis and we have the identity $(1 / 4 \pi) \int d \alpha|\alpha\rangle\langle\alpha|=I_{2 j+1}$, where $I_{2 j+1}$ is the identity operator in the $(2 j+1)$-dimensional Hilbert space spanned by the $|j, m\rangle$ basis states. Any density operator $\rho$ of a spin- $j$ state can be expanded in terms of the $|\alpha\rangle$ in the form of a diagonal representation,

$$
\rho=\int d \alpha P(\alpha)|\alpha\rangle\langle\alpha|
$$

where $P(\alpha)$ is known as the (Glauber-Sudarshan) $P$-function 26] (in general $P(\alpha)$ depends on both $\alpha$ and $\alpha^{*}$, but it is customary to write $P(\alpha)$ for short).

Classically mixing pure states should not increase their quantumness. This principle underlies the well-known definition of classicality in quantum optics [27. In close correspondence, one can therefore define mixed classical spin states as those states that can be written as a classical mixture of spin- $j$ coherent states, i.e. a convex combination of projectors onto spin- $j$ coherent states. This means that a general spin- $j$ state $\rho$ is classical iff there exists a positive function $P(\alpha)$ with which $\rho$ can be written as in Eq. (2) [11. Note that the $P$-function is not unique: indeed, when expanded over spherical harmonics $Y_{K Q}(\theta, \phi)$, only components with $K \leqslant 2 j$ play a role in the integral (2), so that arbitrary spherical harmonics with $K \geqslant 2 j+1$ can be added to $P(\alpha)$ without changing $\rho$ (see an example in [11]). Classical spin- $j$ states are hence those states for which at least one $P$-function is positive. Classical spin- $j$ states form a convex set by definition. Deciding whether a spin- $j$ state is classical or not then becomes a problem of convex optimization (see below). Note that the Wigner function of a spin- $j$ coherent state is not everywhere positive in general, not even for a spin-1/2, see [28]. This is different from the harmonic oscillator, where positivity of the $P$-function implies positivity of $W$.

\section{B. Absolutely classical spin states}

Let $\mathcal{H}_{m}$ be a Hilbert space of dimension $d_{m}$, and $\mathcal{B}\left(\mathcal{H}_{m}\right)$ the space of bounded linear operators on $\mathcal{H}_{m}$. Consider a bipartite physical system with Hilbert space $\mathcal{H}=\mathcal{H}_{m} \otimes \mathcal{H}_{n}$. In [17] the absolute separability problem was introduced: What are the states $\rho \in \mathcal{B}(\mathcal{H})$ such that $U \rho U^{\dagger}$ is separable for all unitary matrices $U \in \mathcal{B}(\mathcal{H})$ ? The problem can also be understood as "separability from spectrum"-problem [18]: Since all $U \rho U^{\dagger}$ have the same spectrum of eigenvalues as $\rho$, it is natural to try to characterize the set of absolutely separable states by conditions on the spectrum. For $n=m=2$, a necessary and sufficient condition is known in terms of a single inequality for the eigenvalues [19]: if $\lambda_{1} \geqslant \lambda_{2} \geqslant \lambda_{3} \geqslant \lambda_{4}$ are the eigenvalues of $\rho$, then it is absolutely separable if and only if $\left[\left(\lambda_{1}-\lambda_{3}\right)^{2}+\left(\lambda_{2}-\lambda_{4}\right)^{2}\right]^{1 / 2} \leqslant \lambda_{2}+\lambda_{4}$. Absolute separability is evidently a stronger condition than separability. For instance a coherent state of two spins- $1 / 2$ is a separable state, but it can become entangled under a general unitary transformation $U \in \mathcal{B}(\mathcal{H})$. More generally, no pure two-qubit state satisfies the 
above inequality, hence any two-qubit absolutely separable state is mixed. The general problem is still open.

In [20] an important step was made by finding the largest ball of separable states (in terms of any $p$-norm, $0 \leqslant p \leqslant \infty)$ centered at the maximally mixed state $\rho_{0}=I_{m} \otimes I_{n} / d$ with $d=m n$. In the Frobenius norm $(p=2)$ its radius is given by $r_{d}=1 / \sqrt{d(d-1)}$, i.e. all $\rho$ with $\left\|\rho-\rho_{0}\right\| \leqslant r_{d}$ are separable, and $r_{d}$ is the largest such constant. In terms of purity this means that $\rho$ is separable if the purity $\operatorname{tr} \rho^{2}$ is less than or equal to $1 /(d-1)$, as was already conjectured in [21]. Although all states within this ball are absolutely separable, there are also absolutely separable states outside this ball [22]. This can be clearly seen in the case $n=m=2$ : it is easy to find examples of states $\rho$ whose distance to $\rho_{0}$ in the Frobenius norm satisfies $\left[\sum_{i}\left(\lambda_{i}-1 / 4\right)^{2}\right]^{1 / 2}>r_{d}=1 / \sqrt{12}$, while the absolute separability condition $\left[\left(\lambda_{1}-\lambda_{3}\right)^{2}+\left(\lambda_{2}-\lambda_{4}\right)^{2}\right]^{1 / 2} \leqslant \lambda_{2}+\lambda_{4}$ is satisfied (for instance $\lambda_{1}=\lambda_{2}=13 / 32$ and $\lambda_{3}=\lambda_{4}=3 / 32$ ). Witnesses for states that are not absolutely separable were introduced in [23].

Here we ask a corresponding question for classicality: what are the spin- $j$ states $\rho \in \mathcal{B}\left(\mathcal{H}_{2 j+1}\right)$ such that $U \rho U^{\dagger}$ is classical for all unitary matrices $U \in \mathcal{B}\left(\mathcal{H}_{2 j+1}\right)$ ? The states that fulfill this criterion will be called "absolutely classical". They are such that no unitary spin- $j$ operator can create quantumness, or equivalently, entanglement among the underlying $N=2 j$ spins $-1 / 2$. We proceed similarly to the approach of [20], i.e. we establish a lower bound on the maximal radius $r_{\max }(j)$ of the ball around the maximally mixed state $\rho_{0}=I_{2 j+1} /(2 j+1)$, in which any state is classical.

\section{Analytical lower bound for $r_{\max }(j)$}

Let $\rho$ be an arbitrary density matrix of a spin- $j$ state. This state can always be written as

$$
\rho(r)=\rho_{0}+r \tilde{\rho}
$$

where $\rho_{0}=I_{2 j+1} /(2 j+1)$, and $\tilde{\rho}=\left(\rho-\rho_{0}\right) /\left\|\rho-\rho_{0}\right\|$ is traceless and normalized so that the (Hilbert-Schmidt or Frobenius) norm of $\tilde{\rho}$ is $\|\tilde{\rho}\|^{2}=\operatorname{tr} \tilde{\rho}^{2}=1$ without restriction of generality. This fixes the scale for the real positive parameter $r$. Therefore the state $\rho(r)$ is at the distance $r$ from the maximally mixed state. The $P$-function of $\rho(r)$, defined through the coherent state representation

$$
\rho(r)=\int d \alpha P(r, \alpha)|\alpha\rangle\langle\alpha|,
$$

can be written

$$
P(r, \alpha)=\frac{1}{4 \pi}+r \tilde{P}(\alpha)
$$

where $1 / 4 \pi$ is the $P$-function of $\rho_{0}$. In order to show that for a given $r$ and arbitrary direction $\tilde{\rho}$ a positive $P$ function can be found, it is enough to consider traceless parts that can be expanded as

$$
\tilde{P}(\alpha)=\sum_{K=1}^{2 j} \sum_{Q=-K}^{K} \tilde{P}_{K Q} Y_{K Q}(\alpha),
$$

where the $Y_{K Q}$ are spherical harmonics and $\tilde{P}_{K Q} \in \mathbb{C}$. Note that more generally $\tilde{P}(\alpha)$ can contain spherical harmonics with arbitrarily large $K$, but any $\rho(r)$ can be represented by a $P$-function that contains values of $K$ only up to $2 j$. Indeed, a given quantum state fixes the components in $P(\alpha)$ up to $K=2 j$ uniquely (see below), whereas the higher ones are arbitrary. Hence we can set them to zero and look for the largest $r$ that still guarantees for all $\tilde{\rho}$ a positive $P(\alpha)$ of the form 6 . We can expand $\tilde{\rho}$ in terms of the irreducible tensor operators $T_{K Q}$ as

$$
\tilde{\rho}=\sum_{K=1}^{2 j} \sum_{Q=-K}^{K} \tilde{\rho}_{K Q} T_{K Q}
$$

Completely analogously, we can also expand $\rho(r)$ and $P(r, \alpha)$ in terms of $T_{K Q}$ and $Y_{K Q}(\alpha)$, respectively:

$$
\begin{gathered}
\rho(r)=\sum_{K=0}^{2 j} \sum_{Q=-K}^{K} \rho_{K Q}(r) T_{K Q}, \\
P(r, \alpha)=\sum_{K=0}^{2 j} \sum_{Q=-K}^{K} P_{K Q}(r) Y_{K Q}(\alpha) .
\end{gathered}
$$

One then immediately finds $P_{K Q}(r)=r \tilde{P}_{K Q}$ and $\rho_{K Q}(r)=r \tilde{\rho}_{K Q}$ for all integer $K \geqslant 1$ and $-K \leqslant Q \leqslant K$. Since $\rho(r)$ is a valid density matrix, the $P_{K Q}(r)$ are related to the $\rho_{K Q}(r)$ by a simple factor [29],

$$
\begin{array}{r}
P_{K Q}(r)=f_{K Q} \rho_{K Q}(r) \forall K, Q, \\
f_{K Q}=(-1)^{K-Q} \frac{\sqrt{(2 j-K) !(2 j+K+1) !}}{2 \sqrt{\pi}(2 j) !},
\end{array}
$$

and hence also

$$
\tilde{P}_{K Q}=f_{K Q} \tilde{\rho}_{K Q}
$$

$\forall K \geqslant 1,-K \leqslant Q \leqslant K$. Cauchy-Schwarz inequality applied to (6) then yields

$$
\begin{aligned}
|\tilde{P}(\alpha)| \leqslant & \left(\sum_{K=1}^{2 j} \sum_{Q=-K}^{K}\left|\tilde{\rho}_{K Q}\right|^{2}\right)^{1 / 2} \\
& \times\left(\sum_{K=1}^{2 j} \sum_{Q=-K}^{K}\left|f_{K Q} Y_{K Q}(\alpha)\right|^{2}\right)^{1 / 2}
\end{aligned}
$$


The normalization of $\tilde{\rho}$ implies

$$
\begin{aligned}
\sum_{K=1}^{2 j} \sum_{Q=-K}^{K}\left|\tilde{\rho}_{K Q}\right|^{2} & =\sum_{K, K=1^{\prime}}^{2 j} \sum_{Q, Q^{\prime}=-K}^{K} \tilde{\rho}_{K Q} \tilde{\rho}_{K^{\prime} Q^{\prime}}^{*} \operatorname{tr} T_{K Q} T_{K^{\prime} Q^{\prime}}^{\dagger} \\
& =\operatorname{tr} \tilde{\rho}^{2}=1,
\end{aligned}
$$

where we have used the orthogonality of the irreducible tensor operators. By noting that $\left|f_{K Q}\right|$ is independent of $Q$ and using the identity

$$
\sum_{Q=-K}^{K}\left|Y_{K Q}(\theta, \varphi)\right|^{2}=\frac{2 K+1}{4 \pi},
$$

we get from 13 that $|\tilde{P}(\alpha)| \leqslant \tilde{P}_{\max }^{(j)}$, with

$$
\tilde{P}_{\max }^{(j)}=\left\{\frac{2 j+1}{8 \pi^{2}}\left[(4 j+1)\left(\begin{array}{l}
4 j \\
2 j
\end{array}\right)-(j+1)\right]\right\}^{1 / 2} .
$$

This implies a lower bound $\tilde{P}(\alpha) \geqslant-\tilde{P}_{\max }^{(j)}$, and hence

$$
P(r, \alpha)=\frac{1}{4 \pi}+r \tilde{P}(\alpha) \geqslant \frac{1}{4 \pi}-r \tilde{P}_{\max }^{(j)}
$$

If the right-hand side is non-negative, so is the left-hand side. Thus if

$$
\begin{aligned}
r \leqslant \frac{1}{4 \pi \tilde{P}_{\max }^{(j)}} & =\left\{(4 j+2)\left[(4 j+1)\left(\begin{array}{l}
4 j \\
2 j
\end{array}\right)-(j+1)\right]\right\}^{-1 / 2} \\
& \equiv \hat{r}_{\max }(j),
\end{aligned}
$$

in the state (3), then the $P$-function, given by $P(r, \alpha)$ in Eq. (5) is positive. Hence, $\rho(r)$ is classical for $r \leqslant$ $\hat{r}_{\max }(j)$. Since $\rho(r)=\rho$ for $r=\left\|\rho-\rho_{0}\right\|$, we have proved that

$$
\left\|\rho-\rho_{0}\right\| \leqslant \hat{r}_{\max }(j) \Rightarrow \rho \in \mathcal{C},
$$

where $\mathcal{C}$ is the set of classical states. The distance $\left\|\rho-\rho_{0}\right\|$ is invariant under conjugation by an arbitrary unitary matrix $U \in \mathcal{B}\left(\mathcal{H}_{2 j+1}\right)$. Hence, if $\rho$ satisfies the inequality in $(19)$, all states $U \rho U^{\dagger}$ verify $\left\|U \rho U^{\dagger}-\rho_{0}\right\| \leqslant \hat{r}_{\max }(j)$ and are thus classical. Therefore $\hat{r}_{\max }(j)$ is a lower bound for the ball size $r_{\max }(j)$.

The Cauchy-Schwarz inequality (13) can be saturated for any given $\alpha$ by choosing $\tilde{\rho}_{K Q}=A f_{K Q} Y_{K Q}(\alpha)$ where $A$ is a proportionality constant such that $\operatorname{tr} \tilde{\rho}^{2}=1$. However, due to the restriction of the $P$-function to (6), with components $K \leqslant 2 j$ only, we do not exhaust all possible $P$-functions. Hence, it may be possible to increase the lower bound of $\tilde{P}(\alpha)$ in $(16)$ by adding components $Y_{K Q}$ with $K>2 j$.

\section{Numerical result for $r_{\max }(j)$}

To test the lower bound (18), we search for nonclassical states that are as close as possible to the maximally mixed state, since each of these states gives an upper bound on the true ball size $r_{\max }(j)$.
To do this, we generate random mixed states $\rho$ from the Hilbert-Schmidt ensemble of matrices $\rho=A A^{\dagger} / \operatorname{tr}\left(A A^{\dagger}\right)$, with $A$ a complex matrix with independent Gaussian entries (see 30 for details). With these states, we construct families of states

$$
\rho_{k}=(1-k) \rho_{0}+k \rho
$$

as function of a parameter $k \in[0,1]$, that interpolate between the maximally mixed state $\rho_{0}$ and the state $\rho$. The task is to find the largest value $k_{\max }$ of $k$, under the condition that $\rho_{k}$ is classical. This can be rewritten as

$$
\max _{k} k \quad \text { s.t. } \quad \rho_{k}=\int d \alpha P(\alpha)|\alpha\rangle\langle\alpha|, P(\alpha) \geqslant 0 .
$$

This problem can be formulated in the form of a linear programming problem, of the form

$$
\max _{x} c^{T} x \quad \text { s.t. } \quad A x=b, x \geqslant 0,
$$

where $x$ is the vector of variables, $c, b$ are real given vectors and $A$ is a real given matrix. These types of optimizations can be solved very efficiently e.g. with an interior-point method [31. Another great property is the existence of a dual problem. If the optimal value of the dual problem coincides with the optimal value of the original problem 22 , i.e. if there is no duality gap the solution is proven to be optimal. We will now explain how to reformulate the problem (21) in the form $(22)$.

Due to Carathéodory's theorem, a positive $P$-function for finite $j$ can always be written as a convex sum of delta functions, so any classical state has the form $\sum_{i=1}^{N} w_{i}\left|\alpha_{i}\right\rangle\left\langle\alpha_{i}\right|$ with $w_{i} \geqslant 0$ and $N \leqslant(2 j+1)^{2}$ (where the number of states needed is reduced by one due to normalization of the state). With this form, $\rho_{k}$ is classical iff there exist $w_{i} \geqslant 0$ with $\sum_{i} w_{i}=1$ and coherent states $\left|\alpha_{i}\right\rangle$ such that

$$
\sum_{i=1}^{N} w_{i}\left|\alpha_{i}\right\rangle\left\langle\alpha_{i}\right|=\rho_{k}
$$

which can be rewritten as

$$
\sum_{i=1}^{N} w_{i}\left|\alpha_{i}\right\rangle\left\langle\alpha_{i}\right|+k\left(\rho_{0}-\rho\right)=\rho_{0} .
$$

This equation can be written as $A x=b$ as in 22, where the vector of variables is given by $x=\left(\left\{w_{i}\right\}_{i=1, \ldots, N}, k\right)$. The vector $b$ is fixed by the maximally mixed state, and the matrix $A$ is constructed from the real and imaginary entries of the left-hand side of Eq. (24). Then with the choice $c=(0, \ldots, 0,1)$ in 22$)$, the problem 21$)$ is in the form of a linear optimization problem.

However, since the $\alpha_{i}$ in (24) are unknown, we generate a large list of uniformly distributed coherent states, of the order of $10^{6}$ many, so that it should be possible to construct almost all classical states by varying the weights 
$w_{i}$. This assumption can be tested by repeating the linear optimization with a new set of random angles and also with an increased number of them. These tests showed that for $j \leqslant 21 / 2$, increaseing the number of random angles beyond $10^{6}$ does not visibly change the results.

We applied this procedure to a list of $n \sim 3000$ different states $\rho$ in 20 for system sizes of up to $j=21 / 2$. The states that maximize $k$ are found at distances

$$
r_{l}=\left\|\rho_{\tilde{k}_{\max }}-\rho_{0}\right\|=\left\|\rho-\rho_{0}\right\| \tilde{k}_{\max }, \quad l=1,2, \ldots, n
$$

from the fully mixed spin- $j$ state, where $\tilde{k}_{\max }$ is the numerical result of the optimization problem $(22),(24)$. Numerically, not all directions $\rho$ can be sampled, and only a finite number of coherent states can be considered. On the one hand, the fact that we can sample only a finite number of coherent states entails that the numerically found $\tilde{k}_{\max }$ for a given $\rho$ is a lower bound of the corresponding exact $k_{\max }$. On the other hand, even if one started with all coherent states, as in the decomposition (21), one would achieve the exact values $\tilde{k}_{\max }=k_{\max }$, but each $r_{l}$, and hence $\tilde{r}_{\max }(j) \equiv \min _{1 \leqslant l \leqslant n} r_{l}$, would still give only an upper bound on the true radius of the ball $r_{\max }(j)$. Therefore, $\tilde{r}_{\max }$ is simply a numerical approximation of $r_{\max }$, but a priori neither a strict upper nor lower bound.

It is worth mentioning that the entangled states closest to the maximally mixed state are not on a straight line with the queen of quantum state, i.e. the state with maximum quantumness for given $j$ [15], except in the $j=1$ case.

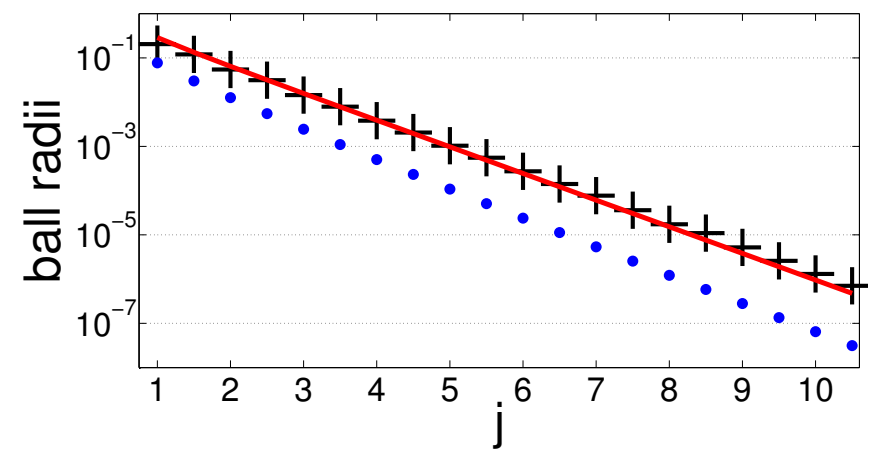

FIG. 1. (Color online). Maximal radius $r_{\max }(j)$ of a ball of classical states centered at the fully mixed state as function of $j$. Blue dots: The value of the lower bound $\equiv \hat{r}_{\max }(j)$, Eq. (18). Black crosses: smallest numerically found distance from the maximally mixed state to a non-classical state. Red line: maximal ball size $1 /\left(2^{j} \sqrt{4^{j}-1}\right.$ ) for arbitrary (not necessarily symmetric) separable states [20]. This function gives an excellent approximation of the numerically found maximal ball size $\tilde{r}_{\max }(j)$ of classical spin- $j$ states, but slightly overestimates it for small $j$.

\section{DISCUSSION}

In Fig. 1 we compare the numerically found $\tilde{r}_{\max }(j)$ and the analytical lower bound $\hat{r}_{\max }(j)$ from Eq. (18) with the radius of the ball of absolutely separable states, $r_{d}=1 /\left(2^{j} \sqrt{4^{j}-1}\right)$ with $d=2^{2 j}[20$. The lower bound $\hat{r}_{\max }(j)$ decays exponentially with $j$. It is still substantially below the numerically found $\tilde{r}_{\max }(j)$, which can be considered close to the exact value $r_{\max }(j)$. Also $\tilde{r}_{\max }(j)$ decays exponentially with $j$, and the ratio between $\tilde{r}_{\max }(j)$ and $\hat{r}_{\max }(j)$ increases only slowly with increasing $j$ over the whole examined range $1 \leqslant j \leqslant 10.5$. The function $1 /\left(2^{j} \sqrt{4^{j}-1}\right)$ agrees with $\tilde{r}_{\max }(j)$ remarkably well over the whole range of $\rho$. However, it is not to be expected that $1 /\left(2^{j} \sqrt{4^{j}-1}\right)$ is the correct result for $r_{\max }(j)$ for at least two reasons: (i.) the fully mixed state in the fully symmetric sector of Hilbert space $I_{2 j+1} /(2 j+1)$ (under exchange of qubits) is not identical to the fully mixed state in the full Hilbert space $\mathcal{H}, I_{2^{2 j}} / 2^{2 j}$, of $N=2 j$ spins- $1 / 2$. Hence, the balls of absolutely separable states and absolutely classical states are not centered at the same point. For example for two spins- $1 / 2$, we have a fully symmetric subspace of $\mathcal{H}$ of dimension 3 (the triplet sector) with the identity matrix $I_{3} \equiv \sum_{m=-1}^{1}|1, m\rangle\langle 1, m|$, whereas the identity in the full $\mathcal{H}$ also contains a projector onto the singlet state $|j=0, m=0\rangle\langle j=0, m=0|$, and has hence to be normalized differently as well, $I_{4} \equiv$ $\sum_{j=0,1} \sum_{m=-j}^{j}|j, m\rangle\langle j, m|$. And (ii.), when minimizing the distance to non-classical states, the relevant set of states is larger without the restriction to symmetric states. From the latter argument one would expect that $1 /\left(2^{j} \sqrt{4^{j}-1}\right)$ underestimated $r_{\max }(j)$, if it were evaluated centered on the same identity. This appears to be correct for large values of $j$ (starting at about $j \geqslant 4$ ), but could there also be due to the numerical uncertainty of the very small value of $r_{\max }(j)$. For small values of $j$, we have rather $\tilde{r}_{\max }(j)<1 /\left(2^{j} \sqrt{4^{j}-1}\right)$. The case $j=1$ is particularly instructive, as there we have a full analytical characterization of the set of classical states [13]. The numerically found value $\tilde{r}_{\max }(j) \simeq 0.2052$ agrees well with the analytical one $1 /(2 \sqrt{6}) \simeq 0.2041$ whereas $1 /\left(2^{j} \sqrt{4^{j}-1}\right)=1 /(2 \sqrt{3}) \simeq 0.288$. Nevertheless, altogether we see that the closest non-classical symmetric state of a spin-j is about as close to the fully mixed state in the symmetric sector as the closest entangled state without any symmetry restrictions to the fully mixed state in the full $2^{2 j}$ dimensional Hilbert space of $N=2 j$ spins- $1 / 2$.

Acknowledgments: DB thanks OG, the LPTMS, and the Université Paris Saclay for hospitality. We thank Peter Braun for useful discussions, and the DeutschFranzösische Hochschule (Université franco-allemande) for support, grant number CT-45-14-II/2015. Ce travail a bénéficié d'une aide Investissements d'Avenir du LabEx PALM (ANR-10-LABX-0039-PALM). 
[1] R. Jozsa and N. Linden, Proc. R. Soc. Lond. A 459, 2011 (2003).

[2] C. H. Bennett, G. Brassard, C. Crepeau, R. Jozsa, A. Peres and W. K. Wootters, Phys. Rev. Lett. 70, 1895 (1993).

[3] A. Harrow, P. Hayden, and D. Leung, Phys. Rev. Lett. 92, 187901 (2004).

[4] D. P. DiVincenzo, D. W. Leung, and B. M. Terhal, IEEE Trans. Inf. Theory 48, 580 (2002).

[5] A. K. Ekert, Phys. Rev. Lett. 67, 661 (1991).

[6] V. Giovannetti, S. Lloyd, and L. Maccone, Science 306, 1330 (2004).

[7] H. Ollivier and W. H. Zurek, Phys. Rev. Lett. 88, 017901 (2001).

[8] L. Mandel, Phys. Scr. T12, 34 (1986).

[9] M. S. Kim, E. Park, P. L. Knight, and H. Jeong, Phys. Rev. A 71, 43805 (2005).

[10] A. M. Perelomov, Commun. Math. Phys. 26, 222-236 (1972).

[11] O. Giraud, P. Braun, and D. Braun, Phys. Rev. A 78, 42112 (2008).

[12] F. Bohnet-Waldraff, D. Braun, and O. Giraud, Phys. Rev. A 93, 012104 (2016).

[13] O. Giraud, P. Braun, and D. Braun, Phys. Rev. A 85, 032101 (2012).

[14] F. Bohnet-Waldraff, D. Braun, and O. Giraud, Phys. Rev. A 94, 42343 (2016).

[15] O. Giraud, P. Braun, and D. Braun, New J. Phys. 12, 063005 (2010).
[16] J. Martin, O. Giraud, P. A. Braun, D. Braun, and T. Bastin, Phys. Rev. A 81, 62347 (2010).

[17] M. Kuś and K. Życzkowski, Phys. Rev. A 63, 32307 (2001).

[18] "Separability from spectrum" - OpenQIProblemsWiki http://qig.itp.uni-hannover.de/qiproblems/15.

[19] F. Verstraete, K. Audenaert, and B. De Moor, Phys. Rev. A 64, 12316 (2001).

[20] L. Gurvits and H. Barnum, Phys. Rev. A 66, 62311 (2002).

[21] K. Życzkowski, P. Horodecki, A. Sanpera, and M. Lewenstein, Phys. Rev. A 58, 883 (1998).

[22] S. Arunachalam, N. Johnston, and V. Russo, Quantum Inform. Comput. 15, 0694, (2015)

[23] N. Ganguly, J. Chatterjee, and A. S. Majumdar, Phys. Rev. A 89, 52304 (2014).

[24] F. T. Arecchi, E. Courtens, R. Gilmore, and H. Thomas, Phys. Rev. A 6, 2211 (1972).

[25] F. Haake, Quantum Signatures of Chaos, (Springer, Berlin, Heidelberg, 2010).

[26] G. S. Agarwal, Phys. Rev. A 24, 2889 (1981).

[27] M. Hillery, Phys. Rev. A 35, 725 (1987).

[28] G. S. Agarwal, Quantum Optics, (Cambridge University Press, Cambridge, 2012).

[29] G. S. Agarwal, Phys. Rev. A 47, 4608 (1993).

[30] K. Życzkowski, K. A. Penson, I. Nechita, and B. Collins, J. Math. Phys. 52, 062201 (2011).

[31] L. Vandenberghe and S. Boyd, SIAM Rev. 38, 49 (1996). 